\title{
PEGylated-PLGA microparticles containing VEGF for long term drug delivery
}

Teresa Simón-Yarza ${ }^{a}$, Fabio R. Formiga ${ }^{a}$, Esther Tamayo ${ }^{a}$, Beatriz Pelacho ${ }^{b}$, Felipe Prosper ${ }^{\mathrm{b}}$, María J. Blanco-Prieto ${ }^{\mathrm{a}^{*}}$

a Pharmacy and Pharmaceutical Technology Department, School of Pharmacy, University of Navarra, Pamplona, Spain

${ }^{\mathrm{b}}$ Hematology Service and Area of Cell Therapy, Clinic Universidad de Navarra, Foundation for Applied Medical Research, University of Navarra, Pamplona, Spain

*Address for correspondence: Maria J. Blanco-Prieto, Department of Pharmacy and Pharmaceutical Technology, School of Pharmacy, University of Navarra, Irunlarrea 1, E-31080 Pamplona, Spain. Tel.: +34 948425600 x 6519; fax: +34 948425649 e-mail: mjblanco@unav.es 


\section{Abstract}

The potential of poly (lactic-co-glycolic) acid (PLGA) microparticles as carriers for vascular endothelial growth factor (VEGF) has been demonstrated in a previous study by our group, where we found improved angiogenesis and heart remodeling in a rat myocardial infarction model (Formiga et al., 2010). However, the observed accumulation of macrophages around the injection site suggested that the efficacy of treatment could be reduced due to particle phagocytosis.

The aim of the present study was to decrease particle phagocytosis and consequently improve protein delivery using stealth technology. PEGylated microparticles were prepared by the double emulsion solvent evaporation method using TROMS (Total Recirculation One Machine System). Before the uptake studies in monocyte-macrophage cells lines (J774 and Raw 264.7), the characterization of the microparticles developed was carried out in terms of particle size, encapsulation efficiency, protein stability, residual poly(vinyl alcohol) (PVA) and in vitro release. Microparticles of suitable size for intramyocardial injection $(5 \mu \mathrm{m})$ were obtained by TROMS by varying the composition of the formulation and TROMS conditions with high encapsulation efficiency (70-90\%) and minimal residual PVA content $(0.5 \%)$. Importantly, the bioactivity of the protein was fully preserved. Moreover, PEGylated microparticles released in phosphate buffer $50 \%$ of the entrapped protein within 4 hours, reaching a plateau within the first day of the in vitro study. Finally, the use of PLGA microparticles coated with PEG resulted in significantly decreased uptake of the carriers by macrophages, compared with non PEGylated microparticles, as shown by flow cytometry and fluorescence microscopy.

On the basis of these results, we concluded that PEGylated microparticles loaded with VEGF could be used for delivering growth factors in the myocardium. 
Keywords: PEG, PLGA, macrophage uptake, VEGF, protein delivery. 


\section{Introduction}

The concept of stealth technology came into being during the World War II in the attempt to escape from radar control. Ever since then, stealth strategy has included two different approaches: the development of radar absorbing paints, and novel designs in terms of shape and size. In the field of drug delivery systems (DDS), this concept has been applied to the ability of these carriers to avoid immunological recognition (Wassef et al., 1991). As in the military context, the shape (Lin et al., 2011), size (Maldiney et al., 2011) and material properties (Essa et al., 2011; Zhu et al., 2011) of the delivery system are crucial.

Recently, some research showing how macrophages have a higher affinity for specific shapes and sizes has been published (Doshi and Mitragotri, 2010). In this paper the authors conclude that particles with a size greater than $4 \mu \mathrm{m}$ suffer less protein adsorption, which is the stage prior to macrophage phagocytosis. Interestingly, when comparing these results with the size distribution of bacteria, they found that most of these have a size between 2 and $3 \mu \mathrm{m}$, which favors their opsonization.

In 1978, Van Oss (Van Oss, 1978) described the phagocytosis process as a surface phenomenon, demonstrating how bacteria that are more hydrophobic than phagocytes readily become phagocytized, whereas bacteria that are more hydrophilic than phagocytes resist phagocytosis. At that time, researchers proposed the surface modification of molecules, by making them more hydrophilic, as a strategy to reduce phagocytic removal. In the 1970s, pegnology, the art of surface-modifying proteins, drugs or DDS by attaching molecules of poly(ethylene glycol) (PEG) was proposed by Abraham Abuchowski and Frank F. Davis (Abuchowski et al., 1977), and this has been applied effectively in protein therapies, obtaining increased stability (Khondee et al., 2011), increased resistance to proteolytic inactivation (Turner et al., 2011), decreased 
immunogenicity (Milla et al., 2011), increased circulatory half-lives (Maleki et al., 2011), and reduced toxicity (Jain, A. and Jain, S.K., 2008), thus improving the delivery and efficacy of proteins. To date, incorporating PEG seems to hold the most promising benefits while showing the lowest harmful effects (Owens and Peppas, 2006) and modified drugs are already on the market, most of which are PEGylated proteins (Pasut et al., 2008), such as interferon alpha (Fried et al., 2002), L-asparaginase (Abuchowski et al., 1984), granulocyte colony-stimulating factor (Tanaka et al., 1991) and uricase (Davis et al., 1981). However, despite the advances in the field of protein therapy, stealth technology is still emerging within the area of DDS. In fact, just one PEGylated delivery system has come onto the market (Knop et al., 2010): a PEGylated liposome containing doxorubicin for the treatment of cancer.

Our group recently published a study in which poly (lactic-co-glycolic) acid (PLGA) microparticles encapsulating the vascular endothelial growth factor (VEGF) were intramyocardially implanted in a ischemia-reperfusion animal model (Formiga et al., 2010). Benefits of the therapy were observed in terms of enhanced angiogenesis and notable reduction of harmful remodeling, but when we studied the continued presence of the particles at the injection site over time, a macrophage accumulation around the particles depot was observed, which could limit the efficacy of the treatment. To overcome this challenge, in the present study our aim was to develop and characterize in vitro PEG-PLGA microparticles loaded with VEGF for their subsequent use in cardiovascular disease. The uptake of VEGF-PEGylated microparticles was studied by flow cytometry and fluorescence microscopy using two different monocyte-macrophage cell lines. Non PEGylated PLGA microparticles were used for comparison. 


\section{Materials and methods}

\subsection{Materials}

Human recombinant VEGF was from R\&D Systems (Minneapolis, MN, USA). PLGA with a lactic:glycolic ratio of 50:50 Resomer $^{\circledR}$ RG 503H (Mw 34KDa), poly[(D,L-lactide-co-glycolide)- co-PEG] diblock Resomer ${ }^{\circledR}$ RGP d 5055 (5\% PEG) and Resomer $^{\circledR}$ RGP d 50105 (10\% PEG) were provided by Boehringer-Ingelheim (Ingelheim, Germany). PEG 400, sodium azide, Rhodamine B isothiocyanate and human serum albumin (HSA) were provided by Sigma-Aldrich (Barcelona, Spain). Dichloromethane and acetone were obtained from Panreac Quimica S.A. (Barcelona, Spain). Poly(vinyl alcohol) (PVA), 88\% hydrolyzed (MW 125000), was from Polysciences, Inc. (WA, USA). Rabbit polyclonal anti-human VEGF-A (clone A-20, sc152) was supplied by Santa Cruz Biotechnology (Santa Cruz, CA, USA). ECL ${ }^{\text {TM }}$ antiRabbit IgG horseradish peroxidase-linked whole antibody was from Amersham Biosciences (Buckinghamshire, UK). Mouse monoclonal anti-rat CD68 was provided by AbD Serotec (Oxford, UK). All the Western blot reagents were purchased from BioRad unless specified in the text.

The murine monocyte-macrophage cells lines J774 and Raw 264.7 were provided by Dr. Latasa (CIMA, University of Navarra). Human umbilical venous endothelial cells (HUVECs) were extracted from umbilical cords from donors, after informed consent according to the guidelines of the Committee on the Use of Human Subjects in Research at the Clinic Universidad de Navarra. CellTiter 96® AQueous One Solution Cell Proliferation Assay (MTS) was obtained from Promega. 


\subsection{Preparation of PLGA and PEG-PLGA microparticles}

VEGF-loaded microparticles were prepared by the double emulsion solvent evaporation method using TROMS (Formiga et al., 2010; Garbayo et al., 2009). Briefly, the organic solution composed of $4 \mathrm{ml}$ of a mixture of dicloromethane/acetone $(3: 1)$ containing $50 \mathrm{mg}$ of Resomer RG503 was injected into the inner aqueous phase, which consisted of $50 \mu \mathrm{g}$ of VEGF in $10 \mathrm{mM}$ phosphate, $50 \mathrm{mM}$ sodium chloride (PBS), $5 \mathrm{mg}$ of HSA and $5 \mu \mathrm{l}$ of PEG400. The primary emulsion $\left(\mathrm{W}_{1} / \mathrm{O}\right)$ was recirculated through the system for $90 \mathrm{sec}$ under a turbulent regime at a flow rate of $38 \mathrm{ml} / \mathrm{min}$. The first emulsion was injected into $20 \mathrm{ml}$ of the external aqueous phase $\left(\mathrm{W}_{2}\right)$ composed of 20 $\mathrm{ml}$ of a PVA solution resulting in the formation of a double emulsion $\left(\mathrm{W}_{1} / \mathrm{O} / \mathrm{W}_{2}\right)$ which was homogenized by circulation through the system for $45 \mathrm{sec}$. The resulting double emulsion was stirred at room temperature for at least 3 hours to allow solvent evaporation and microparticle formation. Finally, microparticles were washed three times with ultrapure water and lyophilized (Genesis 12EL, Virtis). For PEGylated microparticles, $50 \mathrm{mg}$ of a mixture of Resomer ${ }^{\circledR} 503 \mathrm{H}$ and Resomer ${ }^{\circledR}$ RGP d 5055 or Resomer $^{\circledR}$ RGP d 50105 (1:1) were dissolved in the organic phase and microparticles were prepared as described above

The composition of the different phases and TROMS parameters were varied to achieve an adequate particle size (of around $5 \mu \mathrm{m}$ ) for intramyocardial administration, (Formiga et al., 2010).

\subsection{Microparticle characterization}

\subsubsection{Particle size, size distribution and zeta potential}

The mean particle size and size distribution of the microparticles were determined by laser diffractometry using a Mastersizer-S ${ }^{\circledR}$ (Malvern Instruments, 
Malvern, UK). Microparticles were dispersed in distilled water and analyzed under continuous stirring. The results were expressed as mean volume, in micrometers. Samples were measured in triplicate.

The zeta potential was measured using Zetaplus ${ }^{\circledR}$ (Brookhaven instruments, NY, US). Samples were diluted with distilled water and each experiment was repeated three times.

\subsubsection{Residual PVA}

The residual PVA associated with microparticles was determined by a colorimetric method based on the formation of a colored complex between two adjacent hydroxyl groups of PVA and an iodine molecule (Joshi et al., 1979). Briefly, $2 \mathrm{mg}$ of lyophilized microparticles were resuspended in $2 \mathrm{ml}$ of $\mathrm{NaOH} 0.5 \mathrm{M}$ for $15 \mathrm{~min}$ at $60{ }^{\circ} \mathrm{C}$. Each sample was neutralized with $900 \mu \mathrm{l}$ of $1 \mathrm{~N} \mathrm{HCl}$ and the volume was adjusted to $5 \mathrm{ml}$ with distilled water. Next, $3 \mathrm{ml}$ of a $0.65 \mathrm{M}$ solution of boric acid, 0.5 $\mathrm{ml}$ of a solution of $\mathrm{I}_{2} / \mathrm{KI}(0.05 \mathrm{M} / 0.15 \mathrm{M})$ and $1.5 \mathrm{ml}$ of distilled water were added. After $15 \mathrm{~min}$ of incubation, the absorbance of the samples was measured at $690 \mathrm{~nm}$ using an Agilent 8453 UV-visible spectrophotometer (Agilent Technologies, Palo Alto, CA, USA). A standard plot of PVA was prepared under identical conditions. Measurements were performed in triplicate.

\subsubsection{Drug loading and encapsulation efficiency}

The amount of VEGF encapsulated in the microparticles was determined by dissolving $1 \mathrm{mg}$ of microparticles in $50 \mu \mathrm{l}$ of DMSO. VEGF containing samples were diluted in $350 \mu$ of PBS for western-blot analysis. SDS-PAGE was performed onto 
$12 \%$ polyacrylamide gels. Following electrophoresis the proteins were transferred onto nitrocellulose membranes which were then blockaded using 5\% nonfat dried milk in Tris Buffered Saline (TBS) with $0.05 \%$ Tween 20, for $1 \mathrm{~h}$ at room temperature (RT). Membranes were incubated for $2.5 \mathrm{~h}$ at RT with rabbit antihuman VEGF-A antibody (A-20: sc-152, 1:2000 dilution). The bounded antibody was detected with horseradish peroxidase (HRP)-conjugated donkey anti-rabbit IgG antibody (1 h, RT, 1:2000 dilution). Chemiluminiscence detection was performed using LumiLight Plus western blotting substrate (Roche Diagnostics, Mannheim, Germany). The VEGF signal was quantified by densitometry using the Quantity One software (Bio-Rad Laboratories Inc., Munich, Germany). Samples containing defined quantities of VEGF were diluted under the same conditions (PBS and DMSO) and used as standard curve.

\subsection{In vitro release studies}

VEGF loaded microparticles $(1 \mathrm{mg}, \mathrm{n}=3)$ were resuspended in $0.25 \mathrm{ml} \mathrm{PBS} \mathrm{pH}$ 7.4 with $0.02 \%(\mathrm{w} / \mathrm{v})$ sodium azide used as a bacteriostatic agent. Incubation took place under orbital shaking in rotating vials (FALC F200, Falc instruments, Treviglio, Italy) at $37{ }^{\circ} \mathrm{C}$. At predefined times, the tubes were centrifuged $(20000 \mathrm{~g}, 10 \mathrm{~min})$ and the supernatant was removed and frozen at $-80^{\circ} \mathrm{C}$ until it was analyzed by western-blot. The removed solution was replaced with an equal volume of fresh release buffer to maintain sink conditions. Release profiles were expressed in terms of cumulative release and plotted versus time.

\subsection{VEGF bioactivity}

The bioactivity of the VEGF released from the microparticles was evaluated in vitro by determining the proliferative capacity of a human umbilical vein endothelial 
cell (HUVECs) after VEGF treatment. Cells were obtained from human umbilical cord by $0.1 \%$ collagenase II digestion (Jaffe et al., 1973) and expanded in F12K medium (ATCC 30-2004) supplemented with $30 \mu \mathrm{g} / \mathrm{mL}$ endothelial cell growth supplement (ECGS, BD Biosciences), 10\% fetal bovine serum, 1\% sodium heparin and 1\% penicillin/streptomycin.

For the proliferation assay, the cells were plated into 96-well culture plates at a density of $3 \times 10^{3}$ cells/well. After 12 hours, cells were treated with 10 and $25 \mathrm{ng} / \mathrm{ml}$ of free VEGF or released from the microparticles. Culture medium and release medium from non-loaded microparticles were used as control. After $72 \mathrm{~h}$ incubation time under normal culture conditions, proliferation in each group was measured using MTS assay.

\subsection{Uptake of microparticles by macrophages}

The microparticle (PEGylated and non-pelylated microparticles) uptake study was analyzed in two different monocyte-macrophage cell lines by fluorescence microscopy and flow cytometry.

\subsubsection{Fluorescence microscopy}

Fluorescent-labeled microparticles with Rhodamin B isothiocyanate $(0.5 \mathrm{mg} / \mathrm{ml})$ were prepared by adding the marker to the inner aqueous phase. Microparticles were prepared as described above. The uptake of fluorescence particles was evaluated in the monocyte-macrophage $\mathrm{J} 774$ cell line. Cells were plated into a 6 well culture plates at a $70 \%$ confluence in serum free RPMI medium containing $1 \%$ penicillin/streptomycin. Four hours later culture medium (control), Rhodamine B isothiocyanate PLGA or PEGPLGA microparticles were added at a final concentration of $0.33 \mathrm{mg} / \mathrm{ml}$. After 3 hours, culture supernatant containing microparticles was removed and the wells were washed 
three times with PBS. Fluorescence microparticles inside the cells were visualized using an EVOSfl fluorescence microscope (Euroclone, Milan, Italy). The fluorescent signal (corresponding to particle uptake) was quantified using the ImageJ software. Ten fields per well were randomly analyzed (experiments performed in triplicate). The signal emitted was normalized to the cell number in each field.

\subsubsection{Flow cytometry}

For flow cytometry studies, RAW 264.7 cells were seeded at a $30 \%$ confluence in DMEM $10 \%$ serum at $37^{\circ} \mathrm{C}$ and allowed to adhere to the 6 well plate for $48 \mathrm{~h}$. Then the medium was removed and cells were incubated with serum free DMEM for $4 \mathrm{~h}$. PLGA or PEG-PLGA microparticles previously suspended in DMEM were then added $(0.33 \mathrm{mg} / \mathrm{ml})$, whereas the control group received only DMEM. At different time intervals (from 30 minutes up to $3 \mathrm{~h}$ ) the medium was removed, cells were detached, collected and washed three times with PBS. After centrifugation (1500 g, $5 \mathrm{~min})$, the cells were suspended and fixed with $2 \%$ formaldehyde solution for their analysis. Cell complexity or cell granularity was studied by flow cytometry analysis using a BD FACSCalibur flow cytometer for the acquisition of samples. The side scatter (SSC) parameter was recorded as reflecting internal properties of cells (e.g. granularity and refractive index). Data were analyzed using the CellQuest software.

\subsection{Statistics}

Results are expressed as mean $\pm \mathrm{SD}$. Statistical significance was tested on the basis of Student's t test at $95 \%$ confidence intervals. 


\section{Results and discussion}

\subsection{Preparation of PLGA and PEG-PLGA microparticles}

Among the different methods available for protein encapsulation, TROMS was selected because it is a semi-industrial technique capable of encapsulating fragile molecules while maintaining their native properties (Formiga et al., 2010; Garbayo et al., 2008). Since our final goal is to inject the microparticles in the ischemic heart, the aim when preparing the polymeric microparticles was to obtain a size between 5 and $8 \mu \mathrm{m}$, which has been shown to be compatible with intramyocardial administration (Formiga et al., 2010).

During the manufacturing process, size was shown to be affected by polymer composition. The best results, in terms of feasibility, reproducibility and adequate particle size distribution for intramyocardial injection were obtained with the polymer containing $10 \%$ of $\mathrm{PEG}$, and so this polymer was selected for the subsequent experiments

Using the same formulation and maintaining the TROMS parameters to prepare PEGylated and non-PEGylated microparticles, the size was increased for the PEGPLGA co-polymer. Therefore TROMS parameters, mainly first and second emulsion circulation times, were studied in order to achieve PEGylated particles with the desired diameter (Table 1). The circulating times selected were 90 seconds for the first emulsion and 45 seconds for the second emulsion, obtaining a particle size of approximately 6.6 $\mu \mathrm{m}$ (Table 2). Other factors were also studied, such as TROMS needle inner diameter, polymer $\%(\mathrm{w} / \mathrm{v})$ in the organic phase and PVA $\%(\mathrm{w} / \mathrm{v})$ in the external aqueous phase. Selected parameters are resumed in Table 2. 


\subsection{Microparticle characterization}

\subsubsection{Particle size and zeta potential}

As stated above, particles with an average size close to $6 \mu \mathrm{m}$ were obtained for both types of microparticles, which have been demonstrated to be compatible with intramyocardial injection (Formiga et al., 2010).

As shown in Table 3, surface charge values were negative for PEGylated and non-PEGylated particles. However, PEGylated microparticles showed a decreased negative charge $(-8.79 \pm 0.61 \mathrm{mV}$ vs. $-18.10 \pm 0.71 \mathrm{mV})$. This may be attributed to the presence of the PEG chains in the surface of the particle (Essa et al., 2010). Moreover, it has been previously described that a higher PEG chain density on the surface of the particles decreases the mobility of the PEG chains and thus decreases the steric hindrance properties of the PEG layer (Owens and Peppas, 2006). On the other hand, if the PEG concentration is too low, opsonins will attach to the surface and the stealth effect will be decreased. Therefore, in order to achieve an intermediate surface chain concentration between the "mushroom" and the "brush" conformation (low and high PEG concentration respectively), a ratio composition of 1:1 (w/w) of polymers Resomer 503H: Resomer RGP d 50105 was finally selected (Table 3).

\subsubsection{Residual PVA}

PVA contained in the two types of microparticles was less than $2 \%$, being lower for the PEGylated microparticles (Table 3). This lower adsorption of PVA in the surface modified particles could be explained as a consequence of the increased degree of hydrophilicity due to PEG chains, reducing PVA interaction (Essa et al., 2010). In any case, these concentrations are much lower than those reported in the literature for PLA microparticles (Gref et al., 2001) 


\subsection{Drug loading and encapsulation efficiency}

In a previous study, VEGF-PLGA microparticles with high encapsulation efficiency were obtained (Formiga et al., 2010). In the present paper, this growth factor was entrapped into PEG-PLGA microparticles obtaining very high encapsulation values, between 80 and $100 \%$ for both PLGA and PEG-PLGA particles, as determined by western-blot. Moreover, western-blot allowed us to confirm that no degradation of VEGF occurred during the encapsulation process, showing a single characteristic band corresponding to $21 \mathrm{kDa}$ (results not shown).

The ability to quantify proteins by light emitting chemiluminescence detection has been previously studied, highlighting the hotspots which have been taken into account in this research (Dickinson and Fowler, 2002). The results of encapsulation efficiency obtained were also indirectly confirmed in the bioactivity assays (section 3.5). In the cell proliferation study, when treating cells with the protein released from the particles, VEGF concentration was calculated considering the encapsulation values. If cell proliferation is in accordance with the expected VEGF concentration, it is possible to confirm the encapsulation efficiency values, and in this sense the obtained results allow us to consider the western-blot technique as a reliable method to measure the encapsulation efficiency.

\subsection{In vitro release studies}

The amount of VEGF released from the microparticles was measured by an in vitro assay, to confirm that the particles really retain the protein for a period of time and allow a sustained release.

When comparing both types of microparticles, the burst effect was higher for the PEGylated ones, which released approximately $50 \%$ of VEGF within the first 4 hours, 
while $30 \%$ of the entrapped peptide was released from the PLGA particles (Figure 1). Moreover, the plateau was reached after 24 hours for the surface modified particles, whereas for the PLGA particles it occurred after three days. This different release behavior is attributable firstly to the fact that burst effect is mainly due to the protein located in the surface of the particle (Essa et al., 2010; Yoncheva et al., 2009). The presence of PEG chains increases the surface of the particle and, as a consequence, a greater amount of protein attaches to it. Secondly, as PEG chains are hydrophilic, when they are in an aqueous medium, like the release buffer, they are dissolved and this makes it easier for the buffer to get into the matrix, allowing the protein to be released. In any case, it has to be taken into account that a slower protein release is expected in vivo, as previously demonstrated (Blanco-Prieto et al., 2004). The main reason for the slower in vivo kinetics is the low availability of water in the tissue compared with the in vitro conditions, in which the PLGA microparticles are incubated in PBS at $37^{\circ} \mathrm{C}$ and shaken. Moreover, the tissue environment surrounding the microparticles will slow the release of VEGF in vivo.

\subsection{VEGF bioactivity}

VEGF is a growth factor well known for its angiogenic activity (Carmeliet and Jain, 2011). In this sense, it has been demonstrated to promote proliferation of endothelial cells.

In order to confirm that VEGF bioactivity was preserved during the encapsulation/release processes, we tested the ability of VEGF released from the PEGylated particles to stimulate proliferation of HUVEC. Considering protein load and in vitro release profile, cells received the same dose of free VEGF and VEGF released from PEGylated microparticles (10 and $25 \mathrm{ng} / \mathrm{ml})$. Both treatments induced the same 
degree of cell proliferation when compared to control groups (Figure 2). These results allow us to conclude that the presence of PEG in the polymer matrix does not alter the biological properties of the encapsulated VEGF, as it has been previously demonstrated for PLGA microparticles (Formiga et al., 2010). Furthermore, it indicates that this method is useful to encapsulate labile molecules (such as growth factors), retaining their activity, independently of the polymer matrix used.

\subsection{Microparticle macrophage clearance}

Phagocytosis is a process in which macrophages destroy foreign particles in the body. Macrophages (phagocytic cells) are an important part of the immune system and also an important limitation for drug delivery using polymeric microparticles. In order to improve the delivery of VEGF in the ischemic heart, in the present work we prepared VEGF-PEGylated microparticles to avoid the clearance of the microparticles by the phagocytic cells.

\subsubsection{Fluorescence microscopy}

After we incubated J774 cells with particles loaded with Rhodamine B isothiocyanate, the uptake of microparticles by macrophages was clearly observable under fluorescent microscope. Indeed, significant differences in the fluorescent signal inside the cells were detected. In Figure 3 representative images of cells three hours post-treatment with PLGA microparticles (A) and PEG-PLGA microparticles (B) are shown. When quantifying fluorescence we observed a four-fold increase in particle uptake in the case of the PLGA spheres compared to the PEGylated ones (Figure 3 C). These results confirmed the efficacy of the surface modification in the reduction of the macrophage internalization of the PEGylated microparticles. 


\subsubsection{Flow cytometry}

Microparticle uptake by macrophages induces changes in cellular granularity that can be monitored by flow cytometry. Indeed, cells treated with PLGA microparticles showed high granularity levels over incubation time (up to 3 hours), indicating that a large number of particles had been internalized during that period. However, coating the microparticles surface with PEG significantly influenced the uptake of the microparticles by the macrophages. Cells receiving surface modified particles maintained cell complexity in the same way as the non treated cells (control). The differences became significant after incubating the particles for 2 hours in the culture medium (Figure 4). Results obtained using flow cytometry confirmed the observation made by fluorescence microscopy, demonstrating that PLGA microparticles suffer phagocytosis in a more rapid way than PEGylated ones, and consequently confirming that particles have been successfully PEGylated.

\section{Conclusion}

In this study we encapsulated VEGF in stealth microparticles, using a copolymer of PEG and PLGA, with a percentage of PEG adequate to reduce macrophage phagocytosis. PEGylated microparticles with high encapsulation efficiency and suitable size to be implanted in the myocardium were developed. Importantly, the bioactivity of the loaded therapeutic protein was fully preserved. Microparticles whose surface was modified by the incorporation of PEG in the formulation illustrated a significantly decreased uptake by phagocityc cells.

In summary, PEGylation could be a useful approach to obtain growth factorloaded microparticles for myocardial administration, minimizing their local clearance and enhancing the efficacy of the protein therapy in cardiovascular disease. 
Consequently, the next step will be to test the developed microparticles in vivo, in a rat model of myocardial infarction. 


\section{Acknowledgments}

This work was supported by MICCIN PLE2009-0116, PSE SINBAD (PSS 01000002008-1), Caja de Ahorros de Navarra (Programa Tu Eliges: Tu Decides) and the "UTE project CIMA". We thank Dr. Estella-Hermoso de Mendoza for the critical reading of the manuscript.

\section{References}

Abuchowski, A., van Es, T., Palczuk, N.C., Davis, F.F., 1977. Alteration of immunological properties of bovine serum albumin by covalent attachment of polyethylene glycol. J. Biol. Chem. 252, 3578-3581.

Abuchowski, A., Kazo, G.M., Verhoest, C.R., Jr., Van Es, T., Kafkewitz, D., Nucci, M.L., Viau, A.T., Davis, F.F., 1984. Cancer therapy with chemically modified enzymes.

I. Antitumor properties of polyethylene glycol-asparaginase conjugates. Cancer Biochem. Biophys. 7, 175-186.

Blanco-Prieto, M.J., Campanero, M.A., Besseghir, K., Heimgartner, F., Gander, B. 2004. Importance of single or blended polymer types for controlled in vitro release and plasma levels of a somatostatin analogue entrapped in PLA/PLGA microspheres. J. Control. Release 96, 437-448);

Carmeliet, P., Jain, R.K., 2011. Molecular mechanisms and clinical applications of angiogenesis. Nature 473, 298-307. 
Davis, S., Park, Y.K., Abuchowski, A., Davis, F.F., 1981. Hypouricaemic effect of polyethyleneglycol modified urate oxidase. Lancet 2, 281-283.

Dickinson, J., Fowler, S., 2002. Quantification of Proteins on Western Blots Using ECL, in: Walker, J. (Ed.), The Proteins Protocols Handbook, Second ed. Humana Press Inc., Totowa, NJ, pp. 429-437.

Doshi, N., Mitragotri, S., 2010. Macrophages recognize size and shape of their targets. PLoS One 5, e10051.

Essa, S., Rabanel, J.M., Hildgen, P., 2010. Effect of polyethylene glycol (PEG) chain organization on the physicochemical properties of poly(D, L-lactide) (PLA) based nanoparticles. Eur. J. Pharm. Biopharm. 75, 96-106.

Essa, S., Rabanel, J.M., Hildgen, P., 2011. Characterization of rhodamine loaded PEGg-PLA nanoparticles (NPs): effect of poly(ethylene glycol) grafting density. Int. J. Pharm. 411, 178-187.

Formiga, F.R., Pelacho, B., Garbayo, E., Abizanda, G., Gavira, J.J., Simon-Yarza, T., Mazo, M., Tamayo, E., Jauquicoa, C., Ortiz-de-Solorzano, C., Prosper, F., BlancoPrieto, M.J., 2010. Sustained release of VEGF through PLGA microparticles improves vasculogenesis and tissue remodeling in an acute myocardial ischemia-reperfusion model. J. Control. Release 147, 30-37. 
Formiga, F.R., Tamayo, E., Simon-Yarza, T., Pelacho, B., Prosper, F., BlancoPrieto,M.J., 2011.Angiogenic therapy for cardiac repair based on protein delivery systems. Heart Fail. Rev. (In press).

Fried, M.W., Shiffman, M.L., Reddy, K.R., Smith, C., Marinos, G., Goncales, F.L., Jr., Haussinger, D., Diago, M., Carosi, G., Dhumeaux, D., Craxi, A., Lin, A., Hoffman, J., $\mathrm{Yu}, \mathrm{J} ., 2002$. Peginterferon alfa-2a plus ribavirin for chronic hepatitis $\mathrm{C}$ virus infection. N. Engl. J. Med. 347, 975-982.

Garbayo, E., Ansorena, E., Lanciego, J.L., Aymerich, M.S., Blanco-Prieto, M.J., 2008. Sustained reléase of bioactive glycosylated glial cell-line derived neurotrophic factor from biodegradable polymeric microspheres. Eur. J. Pharm. Biopharm. 69, 844-851.

Garbayo, E., Montero-Menei, C.N., Ansorena, E., Lanciego, J.L., Aymerich, M.S., Blanco-Prieto, M.J., 2009. Effective GDNF brain delivery using microspheres-a promising strategy for Parkinson's disease. J. Control. Release 135, 119-126.

R. Gref, P. Quellec, A. Sanchez, P. Calvo, E. Dellacherie, M.J. Alonso, Development and characterization of CyA-loaded poly(lactic acid)-poly(ethylene glycol)PEG microand nanoparticles, Comparison with conventional PLA particulate carriers, Eur. J. Pharm. Biopharm. 51 (2001) 111-118.

Jaffe, E.A., Nachman, R.L., Becker, C.G., Minick, C.R., 1973. Culture of human endothelial cells derived from umbilical veins. Identification by morphologic and immunologic criteria. J. Clin. Invest. 52, 2745-2756. 
Jain, A., Jain, S.K., 2008. PEGylation: an approach for drug delivery. A review. Crit Rev. Ther. Drug Carrier Syst. 25, 403-447.

Joshi, D.P., Lan-Chun-Fung, Y.L., Pritchard, J.G., 1979. Determination of poly(vinyl alcohol) via its complex with boric acid and iodine. Analytica Chimica Acta 104, 153160.

Khondee, S., Olsen, C.M., Zeng, Y., Middaugh, C.R., Berkland, C., 2011. Noncovalent PEGylation by Polyanion Complexation as a Means To Stabilize Keratinocyte Growth Factor-2 (KGF-2). Biomacromolecules 12, 3880-3894.

Knop, K., Hoogenboom, R., Fischer, D., Schubert, U.S., 2010. Poly(ethylene glycol) in drug delivery: pros and cons as well as potential alternatives. Angew. Chem. Int. Ed. Engl. 49, 6288-6308.

Lin, S.Y., Hsu, W.H., Lo, J.M., Tsai, H.C., Hsiue, G.H., 2011. Novel geometry type of nanocarriers mitigated the phagocytosis for drug delivery. J. Control. Release 154, 8492.

Maldiney, T., Richard, C., Seguin, J., Wattier, N., Bessodes, M., Scherman, D., 2011. Effect of core diameter, surface coating, and PEG chain length on the biodistribution of persistent luminescence nanoparticles in mice. ACS Nano 5, 854-862.

Maleki, A., Madadkar-Sobhani, A., Roohvand, F., Najafabadi, A.R., Shafiee, A., Khanahmad, H., Cohan, R.A., Namvar, N., Tajerzadeh, H., 2011. Design, modeling, and expression of erythropoietin cysteine analogs in Pichia pastoris: Improvement of 
mean residence times and in vivo activities through cysteine-specific PEGylation. Eur. J. Pharm. Biopharm. (In press).

Milla, P., Dosio, F., Cattel, L., 2011. PEGylation of Proteins and Liposomes: A Powerful and Flexible Strategy to Improve the Drug Delivery. Curr. Drug Metab. (In press).

Owens, D.E., Peppas, N.A., 2006. Opsonization, biodistribution, and pharmacokinetics of polymeric nanoparticles. Int. J. Pharm. 307, 93-102.

Pasut, G., Sergi, M., Veronese, F.M., 2008. Anti-cancer PEG-enzymes: 30 years old, but still a current approach. Adv. Drug Deliv. Rev. 60, 69-78.

Tanaka, H., Satake-Ishikawa, R., Ishikawa, M., Matsuki, S., Asano, K., 1991. Pharmacokinetics of recombinant human granulocyte colony-stimulating factor conjugated to polyethylene glycol in rats. Cancer Res. 51, 3710-3714.

Turner, K.M., Pasut, G., Veronese, F.M., Boyce, A., Walsh, G., 2011. Stabilization of a supplemental digestive enzyme by post-translational engineering using chemicallyactivated polyethylene glycol. Biotechnol. Lett. 33, 617-621.

Van Oss, C.J., 1978. Phagocytosis as a surface phenomenon. Ann. Rev. Microbiol. 32, 19-39. 
Wassef, N.M., Matyas, G.R., Alving, C.R., 1991. Complement-dependent phagocytosis of liposomes by macrophages: suppressive effects of "stealth" lipids. Biochem. Biophys. Res. Commun. 176, 866-874.

Yoncheva, K., Lambov, N., Miloshev, S., 2009. Modification of biodegradable poly(malate) and poly(lactic-co-glycolic acid) microparticles with low molecular polyethylene glycol. Drug Dev. Ind. Pharm. 35, 449-454.

Zhu, Z., Xie, C., Liu, Q., Zhen, X., Zheng, X., Wu, W., Li, R., Ding, Y., Jiang, X., Liu, B., 2011. The effect of hydrophilic chain length and iRGD on drug delivery from poly(epsilon-caprolactone)-poly(N-vinylpyrrolidone) nanoparticles. Biomaterials 32, $9525-9535$. 


\section{Figure Legends}

Figure 1. In vitro release profiles. VEGF released from PLGA and PEG-PLGA MPs is represented as a $\%$ of the total VEGF load in the particles.

Figure 2. VEGF bioactivity: HUVECs treated with VEGF and VEGF released from the microparticles (MPS-VEGF) at the same concentration (10 and $25 \mathrm{ng} / \mathrm{ml}$ ) proliferate in the same ratio when compared to control groups. ${ }^{*} P<0.05$ and $* * * P<0.001$.

Figure 3. Macrophage uptake studied by fluorescent microscopy. Cells observed under fluorescent microscope after incubating them with PLGA (A) and PEG-PLGA (B) microparticles containing Rhodamine isocyanate. More Rhodamine is visualized inside the cells treated with the PLGA microparticles. This differences are significantly different when quantified (C), indicating that these have been phagocyted in a larger number than those with the PEG chains in the surface. ${ }^{* * *} P<0.001$.

Figure 4. Macrophage uptake studied by flow cytometry. The highest observed cell granularity value (measured as Side Scatter Cell) has been assigned 100\%. Cell complexity increases when cells are treated with PLGA microparticles, whereas cells treated with culture medium or PEG-PLGA microparticles did not have altered complexity three hours after the treatment. ${ }^{*} P<0.05$ and ${ }^{*} * P<0.01$. 